\title{
Aplicación de una alternativa simplificada para modelar la cinética de un proceso de combustión in situ
}

\author{
Alberto Raúl Pinzón Diaz ${ }^{1 *}$, Yohan Harley Pabón Acevedo ${ }^{1}$ Jorge Mario Padilla Reyes², \\ Samuel Fernando Muñoz Navarro ${ }^{1}$ \\ ${ }^{1}$ Grupo de Investigación Recobro Mejorado, Escuela de Ingeniería de Petróleos, Universidad Industrial de Santander, \\ Carrera 27 Calle 9. Bucaramanga, Colombia; \\ ${ }^{2}$ Ecopetrol S.A. Bloque de Ingeniería, Zona Industrial-Campo Casabe, Yondó (Antioquia) \\ *E-mail: alberto.pinzon@correo.uis.edu.co
}

\begin{abstract}
Resumen
La combustión in situ (CIS) es una técnica de recobro mejorado con un gran potencial de aplicación en yacimientos de crudos pesados y extrapesados; sin embargo, su implementación se ha visto limitada por el alto grado de incertidumbre asociado a su desarrollo a escala de campo. Teniendo en cuenta la alta complejidad del proceso con respecto a otras técnicas EOR/IOR, no basta con aplicar un screening binario y realizar pruebas básicas de laboratorio para determinar la factibilidad de aplicarla en un yacimiento determinado, sino que se requiere la realización de estudios adicionales, tales como pruebas de celda cinética, que están orientadas a determinar el comportamiento oxidativo del sistema roca-fluido en estudio, y a su vez, permiten la obtención de un modelo cinético que represente, mediante modelamiento numérico, el desempeño del proceso de CIS obtenido experimentalmente de pruebas de tubo de combustión. Con base en lo anterior, en el presente trabajo, se compararon los resultados obtenidos del ajuste de una prueba de tubo de combustión usando las metodologías Arrhenius y no Arrhenius, evidenciando diferencias significativas en el tiempo de cómputo y en los valores de saturación residual de aceite en el medio poroso después de aplicado el proceso CIS.
\end{abstract}

Palabras clave: Combustión in situ, cinética, simulación numérica, crudo pesado, prueba de tubo de combustión, recuperación mejorada de petróleo.

\section{Simplified alternative to model the kinetics of an in-situ combustion process}

\begin{abstract}
In situ combustion (ISC) is an enhanced oil recovery (EOR) technique with great potential for application in heavy and extra-heavy oil fields; however, its implementation has been limited due to the high degree of uncertainty associated with its development at the field scale. Considering the level of complexity of the ISC process compared to other EOR / IOR techniques, to evaluate the technical feasibility of ISC in a specific reservoir will take far more complex screening methods and laboratory testing. Among the tests required to evaluate ISC include the kinetic cell tests, which are aimed at determining the oxidative behavior of the rock-fluid system under study, and in turn, allow obtaining a kinetic model that represents, by means of numerical modeling, the performance of the ISC process obtained experimentally in combustion tube tests. Based on the above, in the present work, the results obtained from the adjustment of a combustion tube test were compared using the Arrhenius and nonArrhenius methodologies, evidencing significant differences in the computation time and in the residual oil saturation values in the porous medium after the implementation of the ISC process.
\end{abstract}

Keywords: In situ combustion, kinetics, numerical simulation, heavy oil, combustion tube test, enhanced oil recovery (EOR), improved oil recovery (IOR).

Cita: Diaz Pinzón A.R., Pabón Acevedo Y.H., Padilla Reyes J.M. y Muñoz Navarro S.F. (2018). Aplicación de una alternativa simplificada para modelar la cinética de un proceso de combustión in situ. Revista Fuentes: El reventón energético, 16(2), 121-130. 


\section{Introducción}

Mientras que el descubrimiento de nuevos yacimientos petrolíferos decrece, la dependencia de los combustibles fósiles aumenta, haciendo necesario desarrollar técnicas de explotación más eficientes. Dos terceras partes de las reservas remanentes en el mundo son de crudos pesados (10-20 $\left.{ }^{\circ} \mathrm{API}\right)$. El desarrollo de estos yacimientos por métodos convencionales (p.e. depleción, inyección de agua) usualmente es ineficiente debido a la alta viscosidad del aceite. En general para este tipo de yacimientos se recomienda emplear procesos de recobro térmicos (p.e. inyección de vapor, de agua caliente o combustión in situ) donde el principal mecanismo asociado al incremento de la producción de petróleo es la reducción de la viscosidad (Eugono, 2010).

La Combustión In Situ (CIS) es un proceso en el cual se busca llevar a cabo una serie de reacciones exotérmicas entre el oxígeno contenido en el aire inyectado y el hidrocarburo presente en el yacimiento. Producto de estas reacciones, se libera una gran energía en el yacimiento, óxidos de carbono y vapor, que en conjunto provocan una disminución en la viscosidad del hidrocarburo, un incremento en su movilidad, y la combinación de diversos mecanismos de desplazamiento, que derivan en un incremento en la producción y, por ende, del factor de recobro. Contrario a los otros procesos térmicos, la mayoría de la energía requerida en el proceso es generada in situ y es originada por las reacciones entre el oxígeno y el crudo.

Actualmente, el principal desafío para predecir el rendimiento en campo de un proceso de inyección de aire es la caracterización incompleta del proceso de oxidación del crudo y la ausencia de un modelo cinético que permita predecir el comportamiento del proceso bajo diferentes condiciones. Es por esto que desde los años 70's hasta la actualidad diferentes autores como (Burger y Sahuquet, 1972), (Hayashitani, Bennion, Donnelly y Moore, 1978), (Fassihi, 1981), (Adegbesan, Donnelly, Moore, y Bennion, 1986), (Belgrave, Moore, Ursenbach, y Bennion, 1993), (Freitag y Exelby, 2006), (Sequera, Moore, Mehta, y Ursenbach, 2010), (Cinar, 2011), (Khansari, 2014), (Chen, 2012), entre otros, han realizado estudios experimentales acompañados de simulación numérica para obtener un modelo cinético que logre reproducir el comportamiento experimental de la oxidación del petróleo. Con base en lo anterior, Zhu (2011) propuso la metodología WUGI (de sus siglas en inglés "Work-flow based Upscaling for Grid Independence"), o el escalamiento basado en un flujo de trabajo para la independencia del grid, como una alternativa para solucionar algunos de los principales problemas asociados a la simulación del proceso de combustión in situ en simuladores térmicos comerciales. En particular, la metodología WUGI emplea un modelo de reacciones no Arrhenius para representar el proceso de oxidación del crudo.

En este artículo se comparan la metodología Arrhenius propuesta por Cinar (2011) y la metodología no Arrhenius WUGI propuesta por Zhu (2011) para modelar la cinética de un proceso CIS, haciendo énfasis en las ventajas y desventajas que presenta cada una de ellas. Para ello, se ajustó el comportamiento experimental de una prueba de tubo de combustión adelantada sobre un sistema matriz/fluido con las características de un yacimiento de crudo pesado colombiano.

\section{Cinética del proceso de combustión In Situ}

En general, durante la simulación numérica de un proceso de CIS, una vez el frente de combustión alcanza una celda específica, parte del aceite sufre pirolisis generando coque, que es el combustible principal del proceso de combustión, mientras que el resto del aceite fluye hacia la siguiente celda producto de su reducción de viscosidad o por efectos de destilación.

Buscando solucionar el problema de la depositación excesiva de coque como combustible que se presenta cuando se tiene un tamaño de malla de dimensiones de campo, diferentes autores se han enfocado en mejorar el modelamiento del proceso a partir de resultados obtenidos de pruebas de laboratorio (Cinar, 2011), (Zhu, 2011), (Chen, Chen, Moore, Mehta, y Ursenbach, 2014) y (Padilla, 2016). En general, los modelos de reacción empleados para la representación numérica del proceso de CIS se agrupan en modelos de reacción Arrhenius y modelos de reacción no Arrhenius.

\section{Modelo de reacciones Arrhenius}

Como consecuencia de los múltiples componentes que conforman molecularmente el petróleo crudo, se hace difícil describir detalladamente su comportamiento de oxidación y combustión. Una de las estrategias más empleadas para tal fin parte del principio de que, el comportamiento de fases del petróleo está controlado por la información PVT (Presión, Volumen y Temperatura). Por lo tanto, los pseudo componentes de las reacciones en serie y paralelo que tienen lugar durante el proceso CIS para las diferentes fases, son agrupados teniendo en cuenta la información experimental de las pruebas 
PVT. Por otra parte, los coeficientes estequiométricos de los esquemas de reacción son construidos a partir de múltiples balances de masa de los resultados obtenidos en pruebas de tubo de combustión, y validados con el ajuste numérico de los perfiles de temperatura y el historial de producción de gases. En general, se establece que al menos deben ocurrir dos reacciones, una en la que se deposite el combustible y otra en la que se queme (Chen et al., 2014).

En los modelos de reacción basados en la ecuación de Arrhenius, la velocidad de reacción se define como la velocidad de formación de una especie particular por unidad de volumen. Para la mayoría de las reacciones la velocidad de reacción es función tanto de la temperatura como de la concentración de los reactantes. De forma general, la tasa de reacción puede expresarse como el producto entre una constante de velocidad que es función de la temperatura y el modelo de reacción que es función de la concentración como se muestra en la ecuación (1):

$$
\text { Arrhenius }=\frac{d_{C f}}{d t}=A * e^{-E / R T} P_{O_{2}}^{a} * C_{f}^{b}
$$

Aunque han sido diversos los esquemas de reacciones planteados, indudablemente el más empleado desde su planteamiento ha sido el propuesto por Belgrave et al. (1993). Este modelo consta de seis reacciones distribuidas en tres de craqueo, dos de oxidación a bajas temperaturas y una de oxidación a altas temperaturas. El modelo es capaz de reproducir varios de los fenómenos que ocurren durante el proceso de inyección de aire; sin embargo, Cinar (2011) demostró que este esquema, al igual que el propuesto por Crookston, Culham \& Chen (1979), no pueden reproducir adecuadamente el comportamiento experimental de los ensayos RTO. Por lo tanto, para representar el comportamiento experimental en este trabajo se empleará un modelo de reacciones Arrhenius similar al planteado por Cinar (2011).

\section{Modelo de reacciones No Arrhenius}

A escala de yacimiento cuando el frente de reacción pasa por un lugar específico, parte del aceite inicial es quemado (Aprox. 5-10\%) (Sarathi, 1999) y otra parte es desplazada (figura 1). Para representar este comportamiento en los modelos de simulación numérica es necesario escalar la cinética en los modelos a escala de campo, producto de la influencia del tamaño del grid o enmallado en la cinética de las reacciones. Para representar de manera adecuada el proceso de CIS se debe lograr representar que indistintamente del tamaño de las celdas se queme siempre la misma cantidad de aceite.

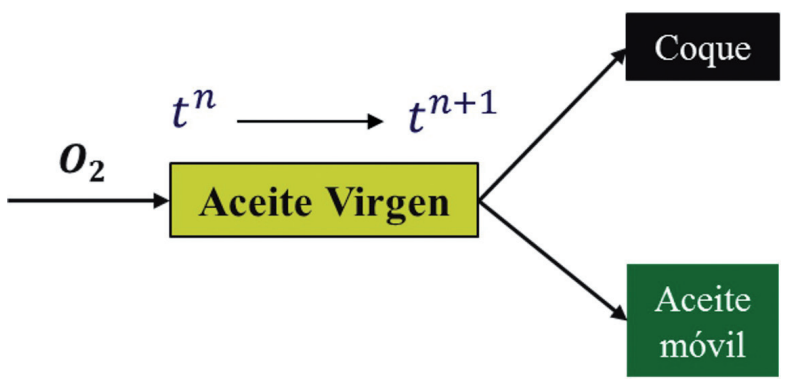

Figura 1. Representación gráfica del escalamiento del proceso de CIS, Zhu (2011)

Considerando que la principal limitante en la simulación numérica del proceso de CIS está asociada a la influencia del tamaño de las celdas sobre las constantes de Arrhenius, empleadas para modelar la cinética de las reacciones en los simuladores térmicos comerciales, el estudio principal considerado en este artículo fue el desarrollado por Zhu (2011). Este estudio propone un nuevo método basado en una cinética no-Arrhenius con enfoque en el escalamiento, usando un modelo subgrid que captura los efectos generales de las reacciones de combustión sobre el flujo y el transporte, buscando dar solución al problema principal del escalamiento, que radica en que el tamaño del frente de reacción es mucho más pequeño (orden de centímetros) que el tamaño que tiene cada bloque del enmallado a escala de yacimiento (orden de metros). Zhu (2011) propuso un modelo cinético que no se rige por las constantes de Arrhenius. Básicamente, sugiere asumir energía de activación de cero para cada una de las reacciones empleadas; de este modo, elimina la dependencia de la temperatura sobre la cinética que es esencialmente el principal problema asociado a la utilización de celdas de gran tamaño en los modelos de simulación. Según esto, la velocidad de reacción para lograr reproducir el comportamiento del proceso de CIS en modelos de simulación numérica a escala de laboratorio y de campo estará dada por la expresión (2):

$$
\text { No-Arrhenius }=\frac{d_{C f}}{d t}=A * P_{O_{2}}^{a} * C_{f}^{b}
$$

Para realizar el escalamiento se emplean las reacciones 3 y 4 :

- Al inicio de la simulación

$$
\mathrm{Oil} \rightarrow(1-\gamma) \mathrm{Oil}_{2}+\gamma \text { Coque }
$$


- Durante la simulación

$$
\text { Coque }+\mathrm{O}_{2} \rightarrow \mathrm{CO}+\mathrm{CO}_{2}+\mathrm{H}_{2} \mathrm{O}
$$

Para calcular el coeficiente estequiométrico $\gamma$, se recomienda que el peso molecular y la densidad del Coque sean cercanos o iguales que los del componente aceite (Oil). Para poder realizar el balance de la reacción en función del factor $\mathrm{x}$ (cantidad de combustible luego de la pirolisis), densidades $\left(\rho_{\text {Coque }}\right.$ y $\left.\rho_{\text {Oil }}\right)$, pesos moleculares $\left(M W_{\text {Coque }}\right.$ y $\left.M W_{\text {Oil }}\right)$ y saturación inicial de aceite $\left(S_{o}\right)$, se requiere el uso de las ecuaciones 5 y 6 , como fue realizado por Zhu (2011) y Glatz (2012):

$$
\begin{gathered}
\gamma=\frac{x * \rho_{\text {Coque }} * M W_{\text {Coque }}}{\rho_{\text {Oil }} * M W_{\text {Oil }} * S_{o}} \\
x=\frac{\text { Volumen de Coque }}{\text { Volumen Poroso }}
\end{gathered}
$$

\section{Representación numérica del comportamiento experimental}

Para la construcción del modelo de simulación numérica, se utilizó la información de una prueba de tubo de combustión realizada para el sistema rocafluido estudiado, a partir de las condiciones operativas mostradas en la tabla 1 . La prueba fue realizada en la unidad de combustión ubicada en laboratorio de inyección de aire del Instituto Colombiano del Petróleo ICP de Ecopetrol S.A.

Con respecto a la malla de simulación, sólo se tuvo en cuenta la sección radial correspondiente al núcleo del empaquetamiento, despreciando el modelado del tubo interno y de las secciones correspondientes a las diferentes capas de aislante. Con base en lo anterior, sólo se ajustaron las temperaturas registradas por las termocuplas internas, considerando que es la zona en donde ocurren las reacciones y los fenómenos fisicoquímicos involucrados en el desarrollo del proceso de combustión.

Tabla 1. Condiciones operativas de la prueba.

\begin{tabular}{|c|c|c|}
\hline Condiciones & Valor & Unidad \\
\hline Modo de la combustión & Seca & NA \\
\hline Presión del ensayo & $\begin{array}{c}(8273,709) \\
(1200)\end{array}$ & $\begin{array}{c}{[\mathrm{KPa}]} \\
{[\mathrm{psi}]}\end{array}$ \\
\hline Temperatura del ensayo & 86 & {$\left[{ }^{\circ} \mathrm{C}\right]$} \\
\hline Caudal de aire & 4 & {$[\mathrm{~L}(\mathrm{~N}) / \mathrm{min}]$} \\
\hline $\mathrm{O}_{2}$ inyectado & 20,94 & {$[\%]$} \\
\hline Tipo de arena & Afloramiento & {$[\mathrm{NA}]$} \\
\hline
\end{tabular}

\section{Definición del enmallado de simulación}

El grid de simulación se muestra en la figura 2, y fue construido en las herramientas computacionales Builder y STARS de la compañía CMG. Para el modelado se optó por realizar un refinamiento en la dirección radial, con el fin de ubicar las termocuplas internas en la misma posición del ensayo experimental y reducir tanto el error numérico asociado, como el error global de balance de masa.

Las propiedades del medio poroso utilizadas para la construcción del modelo de simulación numérica son mostradas en la tabla 2. Los valores de porosidad y permeabilidad son resultado del tamaño de grano utilizado, y afectan el desempeño del proceso con respecto a las variables físicas; sin embargo, estos resultados no son escalables a campo (Sequera et al., 2010), por lo cual no representan ningún inconveniente para la realización de la prueba, considerando que no se afectara la composición de los gases producidos y tampoco los fenómenos químicos que tienen lugar en el proceso.

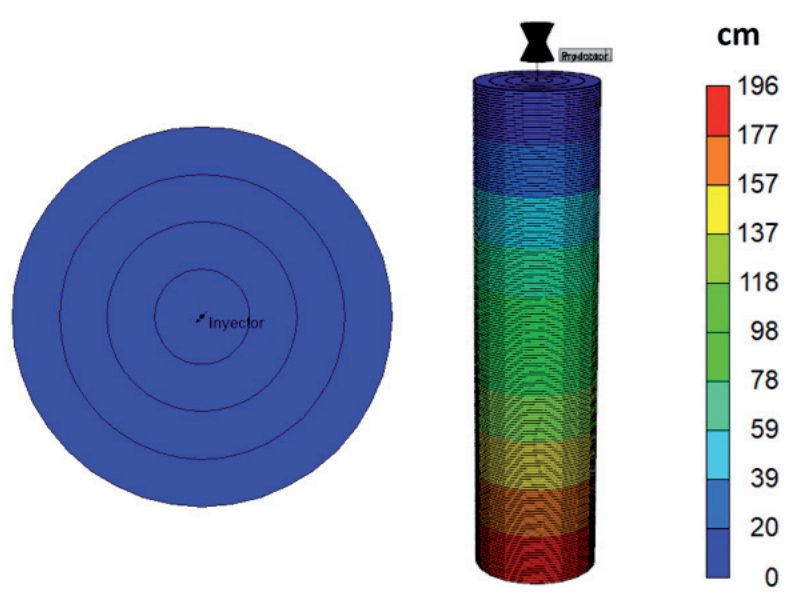

Figura 2. Configuración del grid de simulación.

Tabla 2. Propiedades del modelo de simulación.

\begin{tabular}{|c|c|c|}
\hline Propiedad & Valor & Unidad \\
\hline Porosidad & 40 & {$[\%]$} \\
\hline Permeabilidad i,j,k & 30000 & {$[\mathrm{mD}]$} \\
\hline Saturación de aceite & 71,41 & {$[\%]$} \\
\hline Saturación de gas & 16,35 & {$[\%]$} \\
\hline Caudal de aire & 4 & {$[\mathrm{~L}(\mathrm{~N}) / \mathrm{min}]$} \\
\hline Temperatura inicial & 86 & {$\left[{ }^{\circ} \mathrm{C}\right]$} \\
\hline Presión inicial & $(8273,709)(1200)$ & {$[\mathrm{KPa}]$} \\
{$[\mathrm{psi}]$}
\end{tabular}




\section{Modelos cinéticos}

La correcta selección y definición del modelo cinético, determinará la calidad del ajuste a los resultados experimentales obtenidos; por ello, es necesario definir los parámetros cinéticos que describen el comportamiento oxidativo del crudo candidato a partir de los resultados obtenidos en pruebas de laboratorio. Como se mencionó anteriormente, en esta investigación se realizó una comparación entre el ajuste obtenido a partir de la metodología Arrhenius usando el modelo de Cinar (2011), descrito por las ecuaciones 7, 8, 9 y 10 , y la metodología no Arrhenius a partir de la metodología WUGI de Zhu (2011), descrita por las ecuaciones 11 y 12 .

$$
\begin{gathered}
1.0 \mathrm{Oil}_{1}+4 \mathrm{O}_{2} \rightarrow 12.5 \text { agua }+20 \text { Coque } 1 \\
1 \mathrm{Coque} \rightarrow 1.382353 \text { Coque } 2 \\
\text { Coque } 1+15 \mathrm{O}_{2} \rightarrow 0.61584 \text { agua }+ \\
1.112502 \mathrm{CO}_{2}+0.2416014 \mathrm{CO} \\
\text { Coque } 2+1.4 \rightarrow 0.35552 \text { agua }+1.038335 \mathrm{CO}_{2}+ \\
0.22549471 \mathrm{CO} \\
1.0 \mathrm{Oil}_{1} \rightarrow 0.905892 \mathrm{Oil}_{2}+0.094108 \mathrm{Coque}^{-} \\
\text {Coque }+65 \mathrm{O}_{2} \rightarrow 10.469397 \mathrm{CO}+48.20842 \mathrm{CO}_{2}+ \\
7.7048 \mathrm{H}_{2} \mathrm{O}
\end{gathered}
$$

La tabla 3 muestra el modelo de reacciones Arrhenius seleccionado (Padilla, 2016), incluyendo la estequiometría que fue calculada a partir de los resultados obtenidos en la prueba de tubo de combustión.

Asimismo, el modelo cinético utilizado para realizar

\begin{tabular}{|c|c|}
\hline \multicolumn{2}{|c|}{ Reacción de adición de oxígeno (7) } \\
\hline $\mathrm{A}\left[\mathrm{cm}^{3} / \mathrm{mol}-\mathrm{min}\right]$ & $1.00 \mathrm{E}+09$ \\
\hline $\mathrm{Ea}[\mathrm{KJ} / \mathrm{mol}]$ & $6.50 \mathrm{E}+04$ \\
\hline$\Delta \mathrm{H}[\mathrm{KJ} / \mathrm{mol}]$ & $8.37 \mathrm{E}+05$ \\
\hline $\begin{array}{l}\text { Orden de reacción oxígeno/ } \\
\text { combustible }\end{array}$ & $1.0 / 1.0$ \\
\hline \multicolumn{2}{|c|}{ Reacción de craqueo (8) } \\
\hline $\mathrm{A}\left[\mathrm{cm}^{3} / \mathrm{mol}-\mathrm{min}\right]$ & $5.00 \mathrm{E}+07$ \\
\hline $\mathrm{Ea}[\mathrm{KJ} / \mathrm{mol}]$ & $8.00 \mathrm{E}+04$ \\
\hline$\Delta \mathrm{H}[\mathrm{KJ} / \mathrm{mol}]$ & 0 \\
\hline Orden de reacción del combustible & 1.2 \\
\hline \multicolumn{2}{|c|}{ Reacción de combustión LTO (9) } \\
\hline $\mathrm{A}\left[\mathrm{cm}^{3} / \mathrm{mol}-\mathrm{min}\right]$ & $9.00 \mathrm{E}+14$ \\
\hline $\mathrm{Ea}[\mathrm{KJ} / \mathrm{mol}]$ & $1.20 \mathrm{E}+05$ \\
\hline$\Delta \mathrm{H}[\mathrm{KJ} / \mathrm{mol}]$ & $1.26 \mathrm{E}+06$ \\
\hline $\begin{array}{l}\text { Orden de reacción oxígeno/ } \\
\text { combustible }\end{array}$ & $1.0 / 1.13$ \\
\hline \multicolumn{2}{|c|}{ Reacción de combustión HTO (10) } \\
\hline $\mathrm{A}\left[\mathrm{cm}^{3} / \mathrm{mol}-\mathrm{min}\right]$ & $1.50 \mathrm{E}+14$ \\
\hline $\mathrm{Ea}[\mathrm{KJ} / \mathrm{mol}]$ & $1.40 \mathrm{E}+05$ \\
\hline$\Delta \mathrm{H}[\mathrm{KJ} / \mathrm{mol}]$ & $4.19 \mathrm{E}+05$ \\
\hline $\begin{array}{l}\text { Orden de reacción oxígeno/ } \\
\text { combustible }\end{array}$ & $1.0 / 1.08$ \\
\hline
\end{tabular}
el ajuste de los resultados experimentales a partir de la metodología no Arrhenius es presentado en la tabla 4. Es necesario recordar que según describió Zhu (2011), la reacción de depositación de coque (11) es sintética y sólo tiene por objetivo suministrar de manera espontánea el combustible requerido para la reacción de combustión.
Tabla 3. Modelo Cinético Arrhenius utilizado.

Tabla 4. Modelo Cinético no Arrhenius (WUGI).

Reacción de depositación de combustible (11)

\begin{tabular}{|l|c|}
\hline $\mathrm{A}\left[\mathrm{cm}^{3} / \mathrm{mol}-\mathrm{min}\right]$ & 10 \\
\hline Ea $[\mathrm{KJ} / \mathrm{mol}]$ & 0 \\
\hline$\Delta \mathrm{H}[\mathrm{KJ} / \mathrm{mol}]$ & 0 \\
\hline Orden de reacción Oil1/Oil2 & $1.0 / 1.0$ \\
\hline \multicolumn{2}{|c|}{ Reacción de combustión (12) } \\
\hline $\mathrm{A}\left[\mathrm{cm}^{3} / \mathrm{mol}-\mathrm{min}\right]$ & 100 \\
\hline Ea $[\mathrm{KJ} / \mathrm{mol}]$ & 0 \\
\hline$\Delta \mathrm{H}\left[\mathrm{KJ} / \mathrm{mol} \mathrm{O}_{2}\right]$ & 439.32 \\
\hline Orden de reacción oxígeno/combustible & $1.0 / 1.0$ \\
\hline
\end{tabular}

\section{Resultados}

En un proceso de combustión in situ, existen diversos parámetros que describen el comportamiento del proceso; sin embargo, diferentes autores como Padilla (2016) y Pinzón (2018) demostraron que uno de los comportamientos clave para el ajuste debe ser el perfil 
de temperatura descrito en cada una de las zonas, teniendo en cuenta que es un indicativo de que la liberación energética en la zona se está representando adecuadamente, además de permitir el ajuste de la velocidad del frente de combustión. También, a partir de la representación del comportamiento de la temperatura en la primera zona del tubo de combustión, es posible representar el suministro energético de los calentadores eléctricos, mediante los cuales se alcanza la temperatura de ignición, que para el crudo estudiado es de $350{ }^{\circ} \mathrm{C}$.

Otros comportamientos que deben ser ajustados para garantizar la representatividad del modelo de simulación numérica, son los gases efluentes $\mathrm{CO} \mathrm{y} \mathrm{CO}_{2}$; teniendo en cuenta que, a partir de su cuantificación en la corriente de salida, es posible realizar los múltiples balances de masa que derivan en el cálculo de parámetros de desempeño tales como la cantidad de coque depositado como combustible y el porcentaje de oxígeno que reaccionó.

\section{Modelo Arrhenius}

La figura 3 muestra el comportamiento del perfil de temperatura ajustado a partir de la metodología Arrhenius para diferentes niveles de refinamiento del grid. Es necesario recordar que la validación de un modelo cinético se debe realizar usando un grid altamente refinado, Bazargan et al. (2011), para evitar la dependencia de la cinética con el tamaño del grid, fenómeno descrito por Zhu (2011). También, que las temperaturas tanto internas como externas son monitoreadas en el reactor en 15 puntos diferentes a lo largo de la dirección $\mathrm{k}$, siendo estas temperaturas representativas de cada una de estas 15 zonas, las cuales tienen una longitud aproximada de $12 \mathrm{~cm}$ cada una. Cabe mencionar que el ajuste del comportamiento de temperatura de la zona 1 permitirá establecer que la tasa suministrada por los calentadores para lograr la ignición, además de la liberación energética ocasionada por las reacciones de combustión, fueron representadas de manera adecuada.

A continuación, se muestra la comparación entre los resultados experimentales y 3 escenarios de simulación con 17 celdas, 178 celdas y 356 celdas. Este último escenario, que corresponde a una representación de 0.5 $\mathrm{cm}$ por cada celda en la dirección $\mathrm{k}$, ofrece el mejor ajuste al perfil de la zona 1; y es este escenario el que permite ajustar tanto el alcance de la temperatura de ignición como la generación del frente de combustión posterior, además de no sobreestimar la temperatura máxima alcanzada. También es posible visualizar el efecto que tiene el tamaño del grid sobre la cinética de reacción, teniendo en cuenta que el uso de un grid poco refinado ocasiona que la tendencia descrita se aleje del comportamiento experimental y tienda a superar la temperatura máxima alcanzada, como se evidencia en el escenario de 17 celdas.

Si bien, la utilización de un modelo de simulación robusto permite reducir la incertidumbre a partir de un mayor número de cálculos por cada paso de tiempo, o a partir de la definición de una tolerancia menor en el método de solución, uno de los principales desafíos al realizar simulación numérica de yacimientos es lograr la obtención de predicciones con un nivel de incertidumbre bajo y con un bajo costo computacional. Por ello, es clave obtener un balance entre la incertidumbre de los resultados y el tiempo de cómputo para su obtención. Con base en lo anterior, la tabla 5 muestra una comparación entre los tiempos de cómputo y los errores globales de balance de masa obtenidos de los 3 escenarios elegidos para el modelamiento Arrhenius.

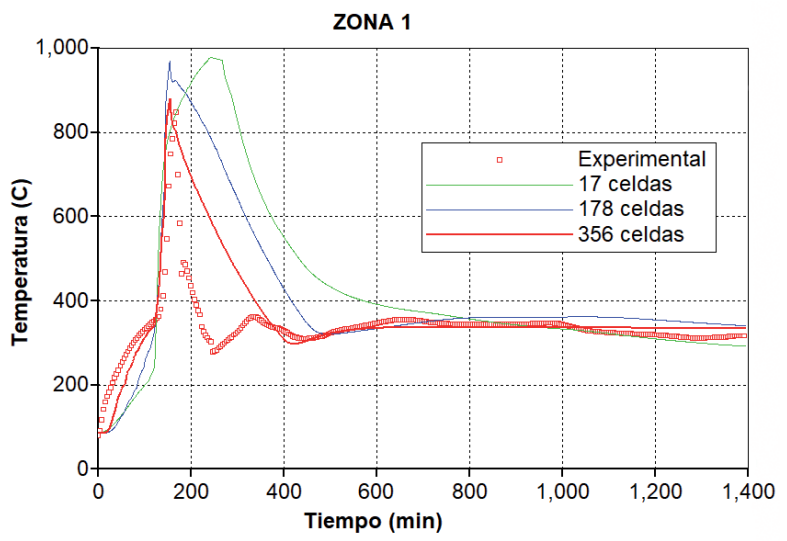

Figura 3. Perfiles de temperatura, modelo Arrhenius. Experimental, 17, 178 y 356 celdas.

Tabla 5. Tiempos de cómputo y errores de balance de masa, escenarios Arrhenius.

\begin{tabular}{|c|c|c|}
\hline Escenario & Tiempo de cómputo & $\%$ Error balance de masa \\
\hline 17 celdas & 0 minutos, 11 segundos & $4 \%$ \\
\hline 178 celdas & 9 minutos, 51 segundos & $3 \%$ \\
\hline 356 celdas & 43 minutos, 8 segundos & $<1 \%$ \\
\hline
\end{tabular}

A partir de los resultados mostrados en la tabla 5, se estableció que de los 3 escenarios considerados para la metodología Arrhenius, el de 356 celdas permitió la obtención del menor error global de balance de masa. Se obtuvo un tiempo de cómputo que, si bien es alto para tratarse de un modelo a escala de laboratorio, es razonable considerando que, para validar el modelo 
cinético seleccionado, se requiere de un alto número de celdas, sin dejar de lado el hecho de que, este valor dependerá de la capacidad de procesamiento del equipo utilizado.

A lo anterior se suma que, el escenario de 356 celdas permitió reproducir el tiempo y la temperatura de ignición, además de mostrar el mejor ajuste al perfil de temperatura descrito en la zona 1.

\section{Modelo no Arrhenius (WUGI)}

De igual manera que para el caso anterior, la figura 4 muestra la comparación entre el comportamiento experimental de la temperatura de la zona 1 y los comportamientos obtenidos para 2 niveles de refinamiento, teniendo en cuenta que al tratarse de una metodología en la cual, la cinética no depende del tamaño de grid, no es necesaria una sensibilización de al menos 3 niveles como en el caso de la metodología Arrhenius.

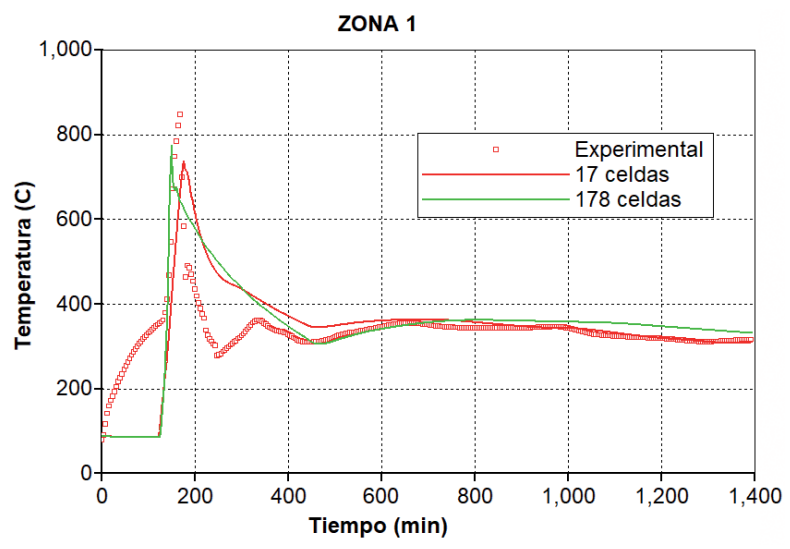

Figura 4. Perfiles de temperatura, modelo WUGI. Experimental, 17 y 178 celdas.

Se evidencia el principio sobre el que se fundamenta la metodología WUGI, al observarse que existe una variación mínima entre los 2 escenarios, y que ambos reproducen el comportamiento de la curva experimental, mostrando que efectivamente existe independencia de la cinética con el tamaño del grid, teniendo en cuenta que un escenario (178 celdas) fue refinado más de 10 veces, en comparación con el otro (17 celdas), sin sobreestimar la temperatura máxima alcanzada, y desviándose ligeramente del comportamiento experimental.

En la figura 4, también se aprecia que, a tiempos tempranos no se reproduce adecuadamente el comportamiento descrito por la curva experimental. Este hecho se debe a que, para este escenario no se incluyó el suministro energético de los calentadores eléctricos como lo describe la curva experimental, considerando que la metodología WUGI no requiere del suministro de energía de una fuente externa para alcanzar la temperatura de ignición, teniendo en cuenta que las reacciones, tanto de depositación de coque, como de combustión son espontaneas, y en cuanto inicia la inyección de aire, se evidencia un incremento en el valor de la temperatura hasta su valor máximo, indicando que el proceso de combustión se está desarrollando y que el frente ha empezado a propagarse.

La tabla 6 muestra la comparación entre los tiempos de cómputo y los errores globales de balance de masa, obtenidos de los 2 escenarios construidos usando la metodología WUGI. A partir de los resultados mostrados, se ratifica el hecho de que ambos escenarios reproducen el comportamiento experimental con baja incertidumbre, al observar que se obtuvieron errores globales en el balance de masa de 3\% para ambos casos. Sin embargo, existe una marcada diferencia con respecto a los tiempos de cómputo, y recordando que se deben buscar resultados representativos a bajo costo computacional, se estableció que el escenario de 17 celdas es la mejor opción para ajustar los comportamientos experimentales con la metodología WUGI.

Tabla 6. Tiempos de cómputo y errores de balance de masa, escenarios WUGI.

\begin{tabular}{|c|c|c|}
\hline Escenario & Tiempo de cómputo & \% Error balance de masa \\
\hline 17 celdas & 0 minutos, 19 segundos & $3 \%$ \\
\hline 178 celdas & 4 minutos, 37 segundos & $3 \%$ \\
\hline
\end{tabular}

\section{Análisis y discusión de resultados}

A partir de los resultados anteriormente mostrados, es posible afirmar que ambas metodologías (Arrhenius y WUGI) permiten representar adecuadamente el comportamiento de la prueba de tubo de combustión. Sin embargo, para determinar las ventajas y desventajas que presenta cada metodología, es necesario el análisis detallado del avance del frente de combustión, el cual puede realizarse a partir del monitoreo de la temperatura, la concentración de oxígeno y la saturación de aceite.

Para establecer dichas comparaciones, se seleccionaron los escenarios de Arrhenius de 356 celdas, Arrhenius de 17 celdas y WUGI de 17 celdas, con base en los comportamientos obtenidos y en los tiempos de cómputo mostrados. 


\section{Temperaturas alcanzadas}

La figura 5 presenta una comparación entre las temperaturas alcanzadas al final de la prueba realizada para los escenarios seleccionados. Se observa que los escenarios Arrhenius 356 celdas y WUGI 17 celdas, muestran comportamientos similares entre sí, además de representar adecuadamente tanto la posición como la temperatura alcanzadas por el frente de combustión al final de la prueba. Sin embargo, el escenario Arrhenius 17 celdas presenta la temperatura del frente de combustión a una menor distancia del pozo inyector de aire (ubicado en la parte superior del reactor), lo que puede indicar que la velocidad del frente de combustión es menor por efecto de una depositación y quema adicional de coque como combustible.

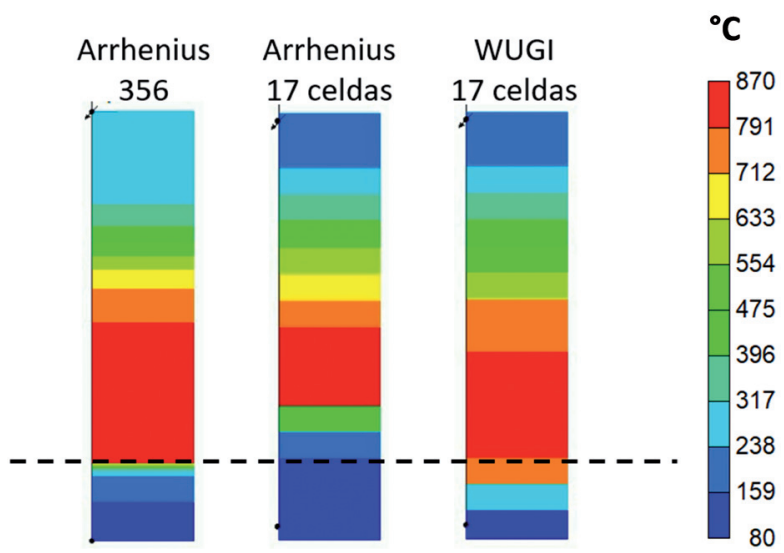

Figura 5. Comparación de temperaturas alcanzadas al final del experimento

\section{Concentración de oxígeno}

La figura 6 muestra la concentración de oxígeno a lo largo del reactor, y nuevamente se evidencia que para el escenario Arrhenius 17 celdas, el frente de combustión no ha alcanzado la misma distancia que en los otros casos, ya que la concentración de oxígeno es de 0.0 en el final del reactor, indicando nuevamente que hay una quema adicional significativa de coque como combustible.

Asimismo, los escenarios Arrhenius 356 celdas y WUGI 17 celdas muestran una leve diferencia en la posición del frente de combustión, lo cual es un indicativo de que en el primer escenario se está consumiendo menor cantidad de coque como combustible en comparación con el último. También es posible visualizar que para los 3 casos hay un consumo total de $\mathrm{O}_{2}$, considerando que el error de balance de cada reacción se ubicó en valores menores a $10^{-5}$, y que no fue incluido $\mathrm{O}_{2}$ como producto. Con base en lo anterior, se puede afirmar que ambos escenarios representan adecuadamente el avance del frente de combustión, analizado a partir del perfil de concentración de oxígeno.

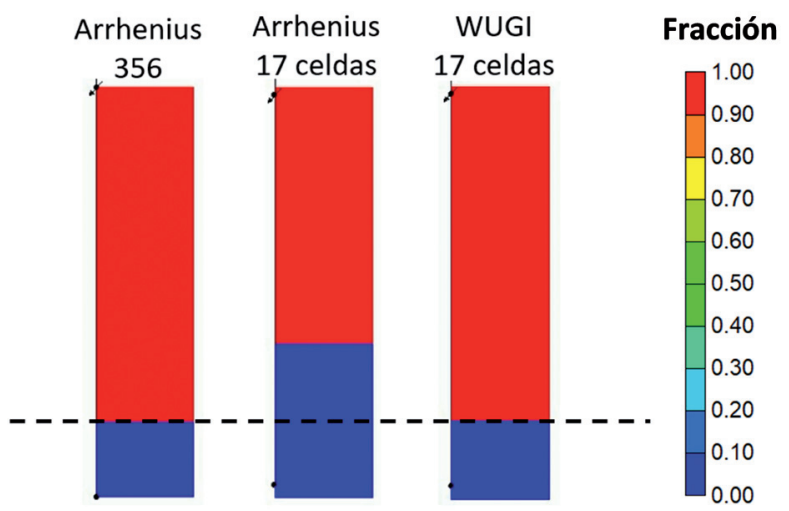

Figura 6. Comparación de concentración de oxígeno al final. del experimento.

\section{Saturación de aceite}

La figura 7 muestra la comparación entre las saturaciones de aceite residual en el reactor obtenidas para los 3 escenarios analizados.

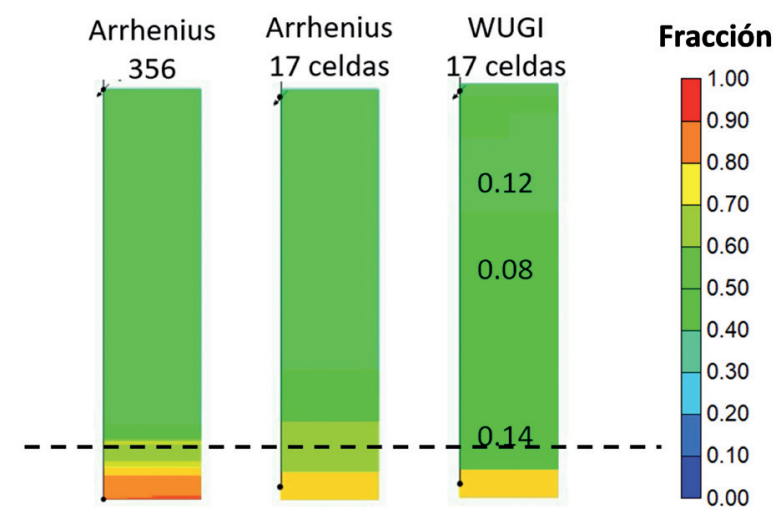

Figura 7. Comparación de saturaciones de aceite residual.

Nuevamente, se evidencia que el comportamiento descrito por el escenario Arrhenius 17 celdas, no reproduce de manera adecuada el comportamiento de la prueba experimental, teniendo en cuenta que hay una saturación de hasta $40 \%$ en la región que ya debió ser barrida. Por su parte, el escenario Arrhenius 356 celdas reproduce adecuadamente el comportamiento, mostrando la saturación residual de aceite de cero detrás del frente de combustión, descrito por Zhu (2011).

Esta propiedad siempre va a ser reproducida adecuadamente por la metodología Arrhenius; sin embargo, en la medida en que las dimensiones del 
grid de simulación se incrementen o el número de celdas se disminuya, el modelo seguirá siendo capaz de representar la saturación residual de aceite de nula, pero aumentando la incertidumbre en los comportamientos de las temperaturas y la composición de los gases efluentes debido al efecto que tiene el tamaño del grid sobre la cinética de reacción, como se evidencia al comparar los escenarios Arrhenius 356 celdas y Arrhenius 17 celdas.

Por su parte, el escenario WUGI presenta una de sus principales limitaciones en cuanto a la representación de los resultados se refiere usando la herramienta computacional STARS de la compañía CMG, por lo que aún deben realizarse esfuerzos conjuntos para desarrollar la manera de reproducir adecuadamente la saturación de aceite residual de cero detrás del frente de combustión, como se muestra a la derecha de la figura 7. Con base en la limitación experimentada, y con la finalidad de reproducir la saturación de aceite residual, se sugiere la modificación del endpoint de saturación de aceite residual, haciéndolo cero durante la construcción de la curva de permeabilidad relativa.

\section{Conclusiones}

A partir del ajuste realizado a una prueba de tubo de combustión para un sistema roca-fluido colombiano, se demostró que el modelado cinético Arrhenius ofreció el mejor ajuste y el error de balance de masa más bajo en comparación con la metodología no Arrhenius WUGI. Sin embargo, no es una metodología adecuada para modelos a escala de campo debido a la dependencia de la cinética con el tamaño del grid.

La metodología WUGI es una alternativa simplificada, en cuanto a tiempo de cómputo se refiere, teniendo en cuenta que logró un ajuste de los resultados de una prueba de tubo de combustión con un error menor a $4 \%$ en masa, en la mitad del tiempo (4 minutos, 37 segundos) de procesamiento que requiere un modelo de simulación numérica con un modelo cinético Arrhenius (9 minutos, 51 segundos).

La principal limitante de la metodología "WUGI", es que asume que la cantidad de combustible depositado "X" no cambia con el tiempo. Con base en lo anterior, se hace necesaria la inclusión del efecto del flux de aire para la determinación de dicha "X", y así reproducir adecuadamente las reacciones de oxidación a baja temperatura que ocurren delante del frente de combustión en presencia de oxígeno.

\section{Agradecimientos}

Los autores expresan su sincero agradecimiento al Instituto Colombiano del Petróleo (ICP) de Ecopetrol S.A., especialmente al equipo de trabajo del laboratorio de inyección de aire. También a la Universidad Industrial de Santander y al Grupo de Investigación Recobro Mejorado por su incondicional apoyo y por permitir el desarrollo de esta investigación.

\section{Referencias}

1. Adegbesan, K. O., Donnelly, J. K., Moore, R. G. y Bennion, D. W. (1986). Liquid phase oxidation kinetics of oil sands bitumen: Models for in situ combustion numerical simulators. AIChE Journal, 32 (8), 1242 - 1252.

2. Bazargan, M., Cinar, M., Glatz, G., Lapene, A., Zhu, Z., Castanier, L., Kovscek, A. R. (2011). A combined experimental and simulation workflow to improve predictability of in situ combustion. SPE 144599.

3. Belgrave, J. D., Moore, R. G., Ursenbach, M. G. y Bennion, D. W. (1993). A comprehensive approach to in situ combustion modeling. SPE Advanced Technology Series. 1 (1), 98 - 107.

4. Burger, J. G., y Sahuquet, G. C. (1972). Chemical Aspects of In Situ Combustion - Heat of Combustion and Kinetics. SPE Journal. 1 (5), $410-422$.

5. Chen, B. (2012). Investigation of in situ combustion kinetics using the isoconversional principle. A Dissertation Ph. D Thesis. Stanford University. Stanford.

6. Chen, X.; Chen, Z.; Moore, R. G.; Mehta, S. A.; y Ursenbach, M. G. (2014). Kinetic Modeling of the In-Situ Combustion Process for Athabasca Oil Sands. SPE 170150.

7. Cinar, M. (2011). Kinetics of crude oil combustion in porous media interpreted using isoconversional methods. A Dissertation Ph. D Thesis, Stanford University. Stanford.

8. Crookston, R., Culham W., and Chen, W. (1979). A numerical simulation model for thermal recovery processes. SPE Journal. 19 (1): 37-58. 
9. Eugono, A. (2010). Numerical simulation of chemical reaction of in situ combustion using SARA fraction. M.Sc. Thesis, Delft University of Technology.

10. Fassihi, M. R. (1981). Analysis of fuel oxidation in In Situ Combustion oil recovery. A Dissertation Ph. D Thesis. Stanford University. Stanford.

11. Freitag, N. P. y Exelby, D. R. (2006) A SARA based model for simulating the pyrolysis reactions that occur in high temperature EOR processes. Journal of Canadian Petroleum Technology. 45 (3), $38-44$.

12. Glatz, G. (2012). Towards Field Scale In-Situ Combustion Simulation. A Dissertation M.Sc. Thesis. Stanford University. Stanford.

13. Hayashitani, M., Bennion, D. W., Donnelly, J. K. y Moore, R. G. (1978). Thermal cracking models for Athabasca oil sands. SPE Annual Fall Technical Conference and Exhibition Proceedings.

14. Khansari, Z. (2014). Low temperature oxidation of heavy crude oil: Experimental study and reaction modeling. A Dissertation Ph. D Thesis. University of Calgary. Calgary.
15. Padilla, J. M. (2016). Desarrollo de un modelo cinético a partir de análisis isoconversional para describir un proceso de combustión in situ. Tesis de Maestría, Universidad Industrial de Santander. Bucaramanga.

16. Pinzón, A. R. (2018) Efecto del flux de aire y las propiedades del medio poroso sobre la cantidad de combustible depositado en un proceso de combustión in situ. Tesis de Maestría, Universidad Industrial de Santander. Bucaramanga.

17. Sarathi, P. S. (1999). In situ Combustion Handbook. Principles and Practices. (BDM Petroleum Technologies) NIPER/BDM-0374. Tulsa, Oklahoma.

18. Sequera, B., Moore, R. G., Mehta, S. A. y Ursenbach, M. G. (2010). Numerical simulation of in situ combustion experiments operated under low temperature conditions. Journal of Canadian Petroleum Technology. 49 (1), $55-64$.

19. Zhu, Z. (2011). Efficient simulation of thermal enhanced oil recovery processes. A Dissertation Ph. D Thesis, Stanford University. Stanford.

Recepción: 04 de octubre de 2018

Aceptación: 04 de diciembre de 2018 